\title{
Cytological Diagnosis of Cylindroma Scalp- A Rare Case Report
}

Dr. Sabina Khan ${ }^{1 *}$, Dr. Sayika Hameed ${ }^{2}$, Dr. Nehal Ahmad MD ${ }^{3}$, Dr. Rubeena Mohroo MD ${ }^{4}$, Dr. Mohd. Jaseem Hassan $\mathrm{MD}^{5}$, Prof. Sujata Jetley MD ${ }^{6}$

\footnotetext{
${ }^{1,5}$ Associate Professor, Department of Pathology, Hamdard Institute of Medical Sciences and Research, Jamia Hamdard New Delhi 110062, India

${ }^{2} 2$ nd year post graduate, Pathology, Hamdard Institute of Medical Sciences and Research, Jamia Hamdard New Delhi 110062, India

${ }^{3}$ Assistant Professor Pathology, Hamdard Institute of Medical Sciences and Research, Jamia Hamdard New Delhi 110062, India

${ }^{4}$ Demonstrator Pathology, Hamdard Institute of Medical Sciences and Research, Jamia Hamdard New Delhi 110062, India

${ }^{6}$ Professor and Head Pathology, Hamdard Institute of Medical Sciences and Research, Jamia Hamdard New Delhi 110062, India
}

DOI: $10.36348 /$ sjpm.2020.v05i05.009

| Received: 07.05.2020 | Accepted: 14.05.2020 | Published: 21.05.2020

*Corresponding author: Dr. Sabina Khan

\section{Abstract}

Cylindromas are rare slow growing benign skin appendageal tumors with eccrine and apocrine differentiation. Multiple cylindromas are commonly genetically inherited and also known as "turban tumor." Here, we have discussed a case of scalp swelling in a male patient having lung cancer, which was clinically suspicious of metastasis. However, cytology revealed many clusters of small round to oval basaloid cells surrounded and attached with magenta coloured acellular hyaline material. The cells had very scanty cytoplasm and dark, round to oval hyperchromatic nuclei without any nuclear membrane irregularity. Cytologic diagnosis of cylindroma was rendered which was subsequently confirmed on histopathology. Cytology of cylindroma should be differentiated from adenoid cystic carcinoma and other benign adnexal tumors like spiradenoma. We wish to report this case as cytology of dermal cylindroma has rarely been dicussed previously in literature and to highlight its cytomorphologic features along with the differential diagnosis.

Keywords: Cylindroma, scalp, cytology.

Copyright @ 2020: This is an open-access article distributed under the terms of the Creative Commons Attribution license which permits unrestricted use, distribution, and reproduction in any medium for non-commercial use (NonCommercial, or CC-BY-NC) provided the original author and sources are credited.

\section{INTRODUCTION}

Cylindromas are rare slow growing benign skin appendageal tumors with eccrine and apocrine differentiation, classified within the family of skin adnexal tumors $[1,2]$. Though cylindroma is classified as apocrine origin but there is controversy regarding its exact origin. The term cylindroma was first used by Billroth in 1859 to describe an orbital tumour. Scalp is the most common site for cylindroma but it also occurs in neck and extremities [1]. Mostly presenting as single lesions, multiplicity is rarely described. Cytodiagnosis of cylindroma has rarely been described in previous literature. Here, we are presenting a case of cylindroma of scalp in a patient diagnosed with Lung carcinoma, which was diagnosed by fine needle aspiration cytology of the lesion.

\section{CASE REPORT}

A 55 year old male presented to Surgery OPD with lobulated swelling on scalp since six months. On examination, there was a firm, non-tender and nodular swelling at left parietal region measuring $4.5 \times 2 \mathrm{~cm}$ which was mobile over underlying skull (Fig-1). The overlying skin appeared to be involved. Imaging features were suggestive of Carcinoma Lung with tiny subpleural nodules $2-3 \mathrm{~mm}$ in both lungs and metastatic deposits in both the kidneys. Patient was clinically diagnosed as Carcinoma lung with cutaneous and systemic metastasis. Scalp swelling was sent for fine needle aspiration cytology to rule out metastatic nodule.

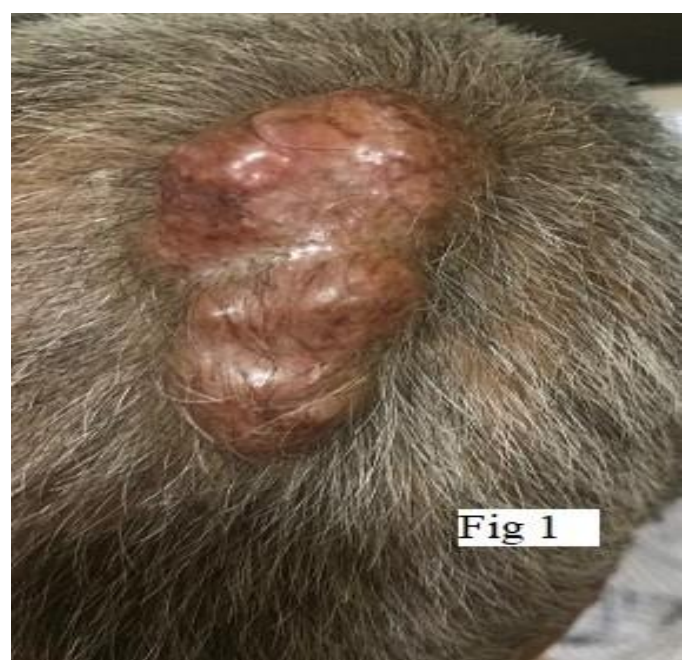

Fig-1: Clinical photograph of firm, nodular swelling on scalp in parietal region 
On aspiration, blood mixed aspirate was obtained. The smears were stained with Giemsa and $\mathrm{H}$ \& E stain. They revealed moderately cellular smears comprising of multiple clusters and discrete small round to oval cells (Fig-2) with darkly stained nuclei, inconspicuous nucleoli and scanty cytoplasm. (Fig-2 inset) Background showed deposits of basement membrane type material appearing as magenta coloured pinkish elongated to globular material (Fig-3). Many of the globules were surrounded by basaloid type of cells. Occasional cells with round to elongated nuclei were noted. No evidence of atypia / malignancy was noted. Based on above cytological features possibility of cylindroma was suggested and histopathological evaluation advised. Histopathology done later confirmed the cytological diagnosis of cylindroma (Fig4).

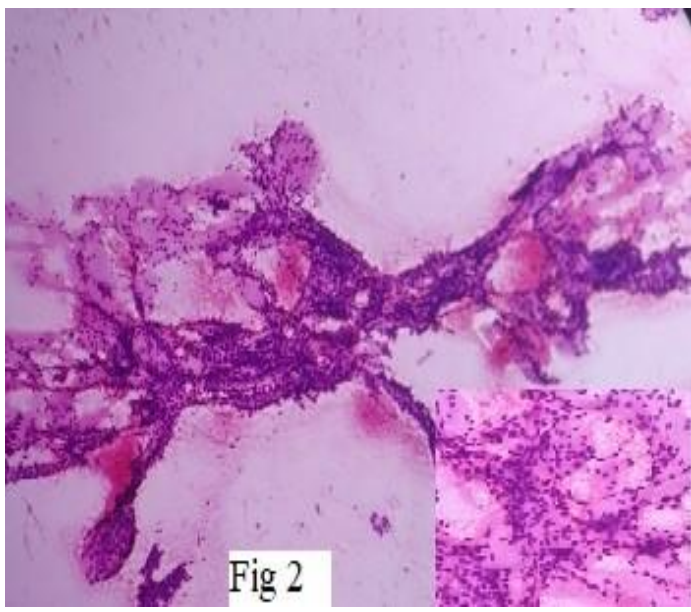

Fig-2: Low power microphotograph showing aggregates and clusters of small, basaloid cells. (H\&E stain, 10x). Inset shows round to oval cells with darkly stained nuclei, inconspicuous nucleoli and scanty cytoplasm (H\&E stain, 40x)

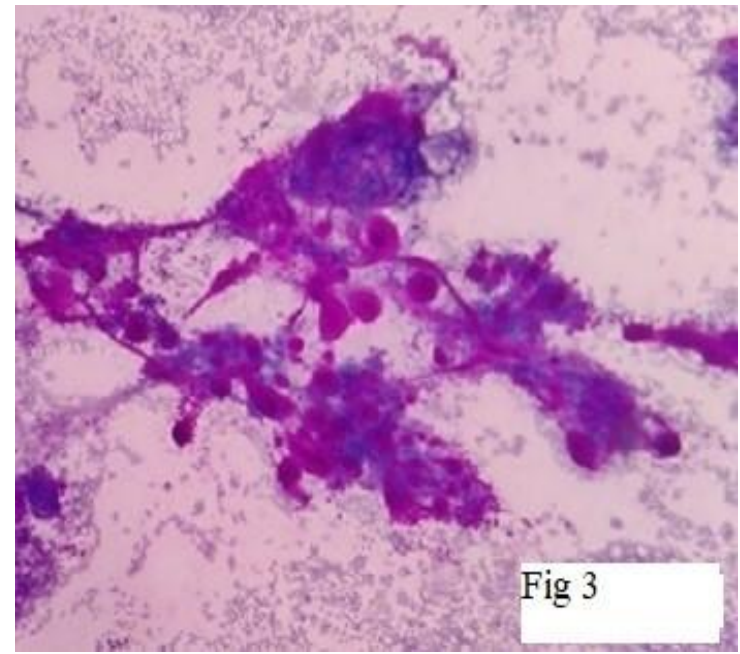

Fig-3: Low power microphotograph showing magenta colored globules of basement membrane material at places surrounded by tumor cells. (H\& E stain, 40x)

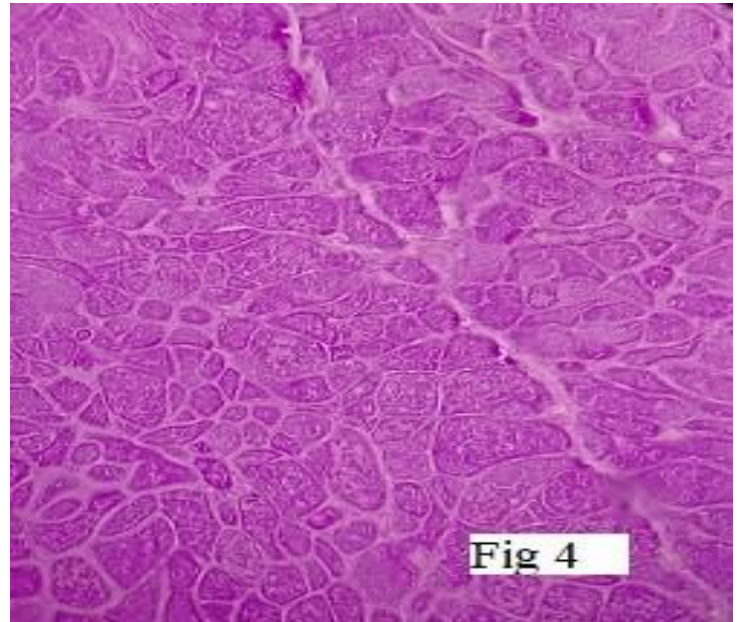

Fig 4: Shows characteristic jigsaw pattern on histopathology separated by basement membrane material. (H\&E stain, 10x)

\section{DISCUSSION}

Cylindroma is a benign skin appendageal tumor. The term arose for its cylindrical shape seen in transversal section. It is predominantly seen in the head and neck region, specially over scalp in middle aged female patients $[1,2]$. Most of the cases are solitary and occur sporadically but multiple lesions may occur in familial cases. The most frequent location for the solitary form is the head and neck. The multiple form usually occurs on the head and neck followed by the trunk and the extremities, and the pubic area. When multiple lesions cover the scalp, the neoplasms are referred to as "turban tumours [3]."

Cylindromas are usually painless, but pain or paraesthesia may occur due to nerve compression. It is generally accepted that bone defects in a cylindroma are signs of malignant transformation. To date, there has been no report on bone destruction associated with the occurrence of a turban tumour [4]. Ultrastructural and immunohistochemical studies indicate its eccrine origin but cylindromas mostly arise in hair follicle rich region mostly scalp, not at eccrine gland rich region like palmo-plantar [2, 5]. Massoumi et al., suggests epithelial hair follicle origin and its exact pathology of differentiation occurs by epithelial-mesenchymal signalling [6].

Cytologically, cylindromas are highly cellular comprised of two types of cells in small clusters around magenta coloured acellular hyaline material as well as in ill formed acinar pattern. The small dark cells have very scanty cytoplasm and dark stained oval to round nuclei with inconspicuous nucleoli. There are few large cells also having relatively moderate cytoplasm and round to oval vesicular nuclei $[1,2,7]$. Very few case reports are available in literature describing the FNAC features of cylindroma.

Cylindroma is a rarely discussed topic in cytology and most of the time, it is diagnosed as benign adnexal tumor. On FNAC, cylindromas are difficult to 
differentiate from adenoid cystic carcinoma [1, 8]. Basaloid cells around the globular hyaline material are found in both the entities. These two lesions are so similar morphologically that they may be indistinguishable on a purely cytologic basis. However, in adenoid cystic carcinoma the neoplastic cells may have moderate to marked cytological atypia, brisk mitotic activity and lack of thickened basement membrane [9]. Another differential diagnosis of cytological diagnosis of cylindroma is eccrine spiradenoma [10]. Cytology of eccrine spiradenoma contains epithelial, myoepithelial cells, and lymphocytes. The epithelial cells are arranged in a rosette like pattern but it lacks magenta coloured hyaline globules [10]. Another entity to be distinguished is chondroid syringoma but typical hyaline- like globules are not seen in such cases [11].

Cylindroma differ histologically from primary cutaneous adenoid cystic carcinoma by the absence of perineural invasion, invasive growth pattern and characteristic histological morphology [12]. Immunohistochemically, the basaloid cells show diffuse positivity with $\mathrm{P} 63$ and larger cells are CK7 positive. Cylindromas demonstrate actin and/or S-100 protein positivity in basal epithelial cells, consistent with myoepithelial differentiation. The organisation of actin and S-100 protein positivity shows that the tumour is differentiated towards the secretory portion of the eccrine sweat gland [13].

\section{ACKNOWLEDGEMENT}

Source of support: Self

Conflict of interest: None, declared by all authors.

Author inclusion criteria: Dr. Sabina was responsible for the literature search, reviewing of manuscript and diagnosis. Dr. Sayika was involved in drafting the article. Dr. Nihal and Dr. Rubeena were involved in microphotography Dr. Jaseem was involved in editing of the manuscript and Dr. Sujata gave the final approval.

\section{CONCLUSION}

Cutaneous cylindromas are uncommon benign adnexal tumours. Through this case report we wish to emphasize on the cytomorphological features of cylindroma and its cytological differential diagnosis as it is a rarely encountered lesion on cytology with very few reports previously described in the literature.

\section{REFERENCES}

1. Gupta, P., Dey, P., \& Bal, A. (2014). Fine needle aspiration cytology of dermal cylindroma. Journal of Cytology/Indian Academy of Cytologists, 31(4), 213-214.

2. Aggarwal, R., Gupta, O., Yadav, Y. K., \& Dogra, S. (2013). Cylindroma of the breast: a rare case report. International journal of trichology, 5(2), 83-85.

3. Kuklani, R. M., Glavin, F. L., \& Bhattacharyya, I. (2009). Malignant cylindroma of the scalp arising in a setting of multiple cylindromatosis: a case report. Head and neck pathology, 3(4), 315-319.

4. Lyon, J. B., \& Rouillard, L. M. (1961). Malignant degeneration of turban tumor of the scalp. Trans $S t$ Johns Hosp Dermatol Soc, 46: 74-77.

5. Klein, W., Chan, E., \& Seykora, J. T. (2005). Tumors of the epidermal appendages. In: Elder, D. E., Elenitsas, R., Johnson, B. L., \& Murphy, G. F., editors. Lever's histopathology of the skin. 9th ed. Ch. 30. Philadelphia, PA: Lippincott Williams and Wilkins; 897-898.

6. Massoumi, R. (2006). Cylindroma as tumor of hair follicle origin. J Invest Dermatol, 126, 1182-1184.

7. Vujhini, S. K., Kolte, S. S., Sushma, Y., \& Satarkar, R. N. (2011). Cytomorphology of solitary cylindroma of the breast. Indian Journal of Pathology and Microbiology, 54(1), 204-205.

8. Wang, N., Leeming, R., \& Abdul-Karim, F. W. (2004). Fine needle aspiration cytology of breast cylindroma in a woman with familial cylindromatosis: a case report. Acta cytologica, 48(6), 853-858.

9. Albores-Saavedra, J., Heard, S. C., McLaren, B., Kamino, H., \& Witkiewicz, A. K. (2005). Cylindroma (dermal analog tumor) of the breast: a comparison with cylindroma of the skin and adenoid cystic carcinoma of the breast. American journal of clinical pathology, 123(6), 866-873.

10. Sinha, A., Pal, S., \& Phukan, J. P. (2014). Fine needle aspiration cytology of eccrine spiradenoma of back: Report of a rare case. Journal of laboratory physicians, 6(2), 130.

11. Daskalopoulou, D., Galanopoulou, A., Statiropoulou, P., Papapetrou, S., Pandazis, I., \& Markidou, S. (1998). Cytologically interesting cases of primary skin tumors and tumor- like conditions identified by fine- needle aspiration biopsy. Diagnostic cytopathology, 19(1), 17-28.

12. Naylor, E., Sarkar, P., Perlis, C. S., Giri, D., Gnepp, D. R., \& Robinson-Bostom, L. (2008). Primary cutaneous adenoid cystic carcinoma. Journal of the American Academy of Dermatology, 58(4), 636-641. 